\title{
Periprosthetic Occult Fractures of the Acetabulum Occur Frequently During Primary THA
}

\author{
Kazuhiro Hasegawa MD, Tamon Kabata MD, PhD, Yoshitomo Kajino MD, PhD, \\ Daisuke Inoue MD, Hiroyuki Tsuchiya MD, PhD
}

Received: 2 June 2016/Accepted: 19 October 2016/Published online: 31 October 2016

(C) The Association of Bone and Joint Surgeons (B) 2016

\begin{abstract}
Background Periprosthetic fractures of the acetabulum occurring during primary THA are rare. Periprosthetic occult fractures are defined as those not identified by the surgeon during the procedure which might be missed on a routine postoperative radiograph. However, it is unclear how frequently these fractures occur and whether their presence affects functional recovery.

Questions/Purposes In this study, using routine CT scans that were obtained as part of another primary hip arthroplasty study protocol, we retrospectively assessed (1) the prevalence of occult fractures of the acetabulum occurring during primary THA, (2) the location of occult fractures of the acetabulum during THA, and (3) risk factors contributing to such occult fractures.
\end{abstract}

Each author certifies that he or she, or a member of his or her immediate family, has no funding or commercial associations (eg, consultancies, stock ownership, equity interest, patent/licensing arrangements, etc) that might pose a conflict of interest in connection with the submitted article.

All ICMJE Conflict of Interest Forms for authors and Clinical Orthopaedics and Related Research ${ }^{\mathbb{R}}$ editors and board members are on file with the publication and can be viewed on request.

Clinical Orthopaedics and Related Research ${ }^{\mathbb{R}}$ neither advocates nor endorses the use of any treatment, drug, or device. Readers are encouraged to always seek additional information, including FDAapproval status, of any drug or device prior to clinical use. Each author certifies that his or her institution approved the human protocol for this investigation, that all investigations were conducted in conformity with ethical principles of research, and that informed consent for participation in the study was obtained.

K. Hasegawa, T. Kabata ( $)$, Y. Kajino, D. Inoue, H. Tsuchiya Department of Orthopaedic Surgery, Graduate School of Medical Science, Kanazawa University, 13-1 Takaramachi, Kanazawa, Ishikawa 920-8641, Japan

e-mail: tamonkabata@yahoo.co.jp
Methods Between 2004 and 2013, our institute performed 585 primary THAs (cementless or hybrid) in 494 patients with DICOM pre- and postoperative CT; during the period in question, all patients undergoing THA underwent $\mathrm{CT}$ before and after surgery. Preoperative CT images were taken as part of a CT-based three-dimensional templating software and navigation system. Postoperative CT images were taken an average of 1 week after surgery as part of a different protocol to evaluate cup position, restoration of leg length and offset, volume of postoperative hematoma to assess anticoagulation effects after THA, and fractures that were not found on routine postoperative radiographs (which we defined as occult fractures). Patients with a history of prior pelvic osteotomy, trauma, and infection were excluded (88 patients/99 hips); 406 patients (102 males and 304 females; 486 hips) form the basis of this report. The mean age of the patients was $60 \pm 11$ years, with a mean BMI of $23 \pm 4 \mathrm{~kg} / \mathrm{m}^{2}$. The mean followup of the patients with periprosthetic fracture of the acetabulum was $58 \pm 28$ months (range, 12-131 months). Potential risk factors for occult acetabular fracture including age, sex, BMI, preoperative diagnosis, additional dome screw fixation, composition and size of each cup, and acetabular design were examined in multivariate analysis. Acetabular component designs were categorized as true hemispheric, peripheral self-locking, and elliptical; during the period in question the indications for each cup design were based on the brand of stem used. Comparison between preoperative and postoperative CT images was done to detect the fractures. Patients with fractures identified during surgery were treated with additional dome screw fixation and a 3-week period of nonweightbearing. Patients with occult fractures in this series did not receive additional treatment as we had confirmed secure fixation of the cup during surgery. 
Results Occult fractures occurred in 41 hips (8.4\%); periprosthetic fractures of the acetabulum were seen during surgery in an additional two hips $(0.4 \%)$. The superolateral wall was the most frequent location for occult fractures of the acetabulum. After controlling for relevant confounding variables, only the use of peripheral self-locking cups was associated with an increased risk of occult fracture (odds ratio [OR], 2.6 compared with hemispheric cups; 95\% CI, 1.2-5.6; $\mathrm{p}<0.05$ ). All patients with occult fractures showed bone ingrowth fixation at the final followup, without any additional surgical intervention.

Conclusions Periprosthetic occult fractures of the acetabulum may occur relatively frequently during press-fit impaction. We observed a higher rate of fractures associated with the use of peripheral self-locking components. Occult acetabular fractures not detected on routine postoperative plain films may be ignored if secure fixation of the cup has been confirmed during the operation.

Level of Evidence Level III, therapeutic study.

\section{Introduction}

The use of cementless acetabular components in THA has become more common during the past 20 years [1,29]. Today, many THAs performed in North America use cementless acetabular components [28]. Generally, there are two methods of inserting the cementless socket: lineto-line reaming of the acetabulum and underreaming the acetabulum with press-fit impaction of the component. The latter method, underreaming and press-fit impaction of the component with or without additional screw fixation, has become the most popular fixation technique [11]. With this technique, recognizable intraoperative fractures of the acetabulum sometimes occur during impaction, but such fractures during primary arthroplasty are rare $[6,7,12,23]$. The rate of intraoperative fracture of the acetabulum during primary THA has been reported as $0.4 \%$ [12], whereas the rate of recognizable periprosthetic fractures of the femur with cementless femoral components in primary THA is generally less than 5\% $[3,17,21,24,27]$. Plain radiography alone may not show a periprosthetic fracture of the acetabulum immediately after occurrence; MRI or reconstructive CT frequently is required to confirm the fracture.

As part of other study protocols that obtained preoperative and postoperative CT images on a large number of primary THAs for templating and computer navigation purposes $[10,14-16,18]$, we routinely recognized postoperative acetabular fractures on these images and thought that the incidence of these fractures was unexpectedly high. Previous reports have indicated that underreaming or oversizing the cup (insertion in rigid bone), osteoporosis, and a preoperative diagnosis of rheumatoid arthritis were risk factors for periprosthetic fractures of the acetabulum which were detected during surgery and with postoperative radiographs $[6,7,14,20,30]$. These fractures also occasionally are diagnosed when a patient reports groin pain during the weeks after surgery [25]. As shown in prior studies, fixation of the cup is critical when periprosthetic acetabular fractures are seen at the time of surgery $[8,13]$. However, periprosthetic occult fractures may occur during press-fit cup impaction, go unrecognized during the procedure, and are undetectable on routine postoperative radiographs.

We therefore assessed (1) the prevalence of occult fractures of the acetabulum occurring during primary THA, (2) the location of occult fractures of the acetabulum during THA, and (3) risk factors contributing to such occult fractures.

\section{Methods}

This study was retrospective. Between February 2004 and May 2013, all 494 patients undergoing 585 primary THAs (cementless or hybrid) performed at our institute underwent DICOM pre- and postoperative CT imaging. No THAs performed during this time were excluded from this imaging protocol. The $\mathrm{CT}$ imaging was performed during this time primarily for three-dimensional (3-D) CT-based templating and to confirm cup position after navigationassisted THA. Exclusion criteria for patients in this study included prior history of pelvic osteotomy, trauma, and infection (88 patients/99 hips); the study population thus ultimately included 486 hips (406 patients; 102 males and 304 females). The mean age of the patients was $60 \pm 11$ years (range, 17-87 years), and the mean BMI was $23 \pm 4$ $\mathrm{kg} / \mathrm{m}^{2}$ (range, $14.3-38.0 \mathrm{~kg} / \mathrm{m}^{2}$ ). Preoperative diagnoses included osteoarthritis (OA) in 374 hips, rapidly destructive coxarthropathy in 11 hips, rheumatoid arthritis in 20 hips, osteonecrosis of the femoral head in 69 hips, and other in 12 hips. Acetabular component designs were categorized as true hemispheric, peripheral self-locking, and elliptical cups. Elliptical cups have a peripheral flare and peripheral self-locking cups are structured such that the rim is $1.8 \mathrm{~mm}$ larger than the true cup diameter (Fig. 1). True hemispheric cups $(\mathrm{n}=222)$ included Trilogy ${ }^{\mathbb{R}}$ (ZimmerBiomet, Warsaw, IN, USA), Trident ${ }^{\mathbb{R}}$ hydroxyapatite (HA) hemispheric (Stryker, Mahwah, NJ, USA), ADEPT ${ }^{\circledR}$ (DePuy Synthes, West Chester, PA, USA), AHFIX ${ }^{\circledR}$ Q3 (Kyocera Medical, Osaka, Japan), AMS ${ }^{\circledR}$ HA (Kyocera Medical), BHR ${ }^{\mathrm{TM}}$ (Smith \& Nephew, London, UK), and Pinnacle $^{\circledR}$ (DePuy Synthes) designs. Peripheral self-locking cups $(n=261)$ included Trident ${ }^{\mathbb{R}}$ PSL ${ }^{\mathbb{R}}$ HA peripheral self-locking (Stryker) and TriAD PSL ${ }^{\circledR}$ HA (Stryker). 


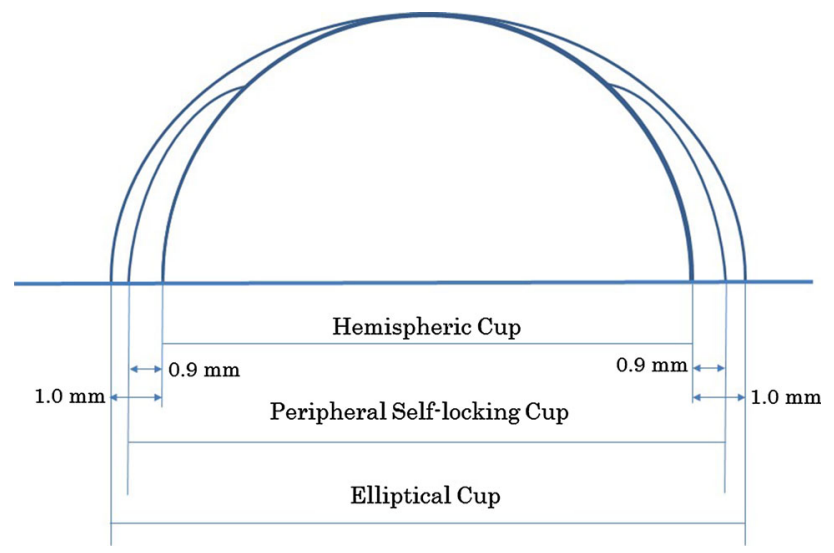

Fig. 1 The diagram shows acetabular component designs of hemispheric, peripheral self-locking, and elliptical cups. Elliptical cups have a peripheral flare $2 \mathrm{~mm}$ larger than a cup diameter and peripheral self-locking cups have a rim that is $1.8 \mathrm{~mm}$ larger than the cup diameter.

Elliptical cups (three) were exclusively Trabecular Metal ${ }^{\mathrm{TM}}$ (Zimmer-Biomet). Of the cups used in the current study, the Trabecular $\mathrm{Metal}^{\mathrm{TM}}$ cup is made of tantalum, the $\mathrm{ADEPT}^{\circledR}$ and $\mathrm{BHR}^{\mathrm{TM}}$ cups are made of $\mathrm{CoCr}$, and the others are made of titanium alloy. Cups were selected based on the manufacturer of the stem used. For femoral implants, the implant that best fit the morphologic features of each patient's medullary cavity was selected by preoperative 3-D templating. We then used a cup whose manufacturer was the same as that of the implant on the femur side. The peripheral self-locking cup was the first choice when we used Trident ${ }^{\circledR}$ stems. Each design was analyzed for fracture risk. Additionally, we conducted univariate analysis of the risk factors for acetabular periprosthetic fractures found during surgery and the occult fractures. These included sex, age, BMI, and preoperative diagnosis.

During preoperative planning, radiographic transparent cup templating and CT-based 3-D templating were performed for all patients to evaluate the position of the cup and its osseous coverage by acetabular host bone. We planned in principle for placement at the level of the true acetabulum. If positioning at that level did not appear to obtain sufficient bony coverage, the cup position was elevated superiorly (not superolaterally) until the level at the center of the cup was covered by host bone (cup centeredge angle became greater than $0^{\circ}$ ) [26]. The angle of the cup was aimed at an anatomic inclination of $40^{\circ}$ and anteversion of $20^{\circ}$. All procedures were performed by the senior surgeon (TK), primarily using a posterolateral approach. All peripheral self-locking cups were press-fit after reaming to the same size as the cup diameter $(1.8 \mathrm{~mm}$ underreaming for the rim diameter), and all hemispheric cups were press-fit after 1 to $2 \mathrm{~mm}$ underreaming based on the patient's bone quality. We judged the bone quality by sex, age, medical history, preoperative images, and perioperative findings such as resistance to reaming. Peripheral self-locking cups generally were used as the first-choice acetabular implant in primary THA with Stryker stems because we believed we could obtain excellent initial fixation with this design. However, if the trial cup appeared too tight on introduction into the acetabulum after reaming to the same size as the planned peripheral self-locking cup diameter, we usually changed to a hemispheric cup; this might have contributed to comparative fracture prevention but also attendant selection bias.

After press-fit fixation, one to two dome screws were inserted if needed. In our institution, the surgeon (TK) routinely inserted screws regardless of the rigidity of pressfit when full coverage of the cup was not obtained by the host bone, as in cases of dysplasia. However, in primary $\mathrm{OA}$ and osteonecrosis of the femoral head, a screwless approach was used when press-fit fixation was judged to be sufficient. With the use of a one-piece metal-on metal cup such as a BHR $^{\mathrm{TM}}$ or $\mathrm{ADEPT}^{\circledR}$ cup, a screwless approach was performed by necessity. In the case of less-than-ideal fixation with press-fit techniques, we used screws.

In our institute, in addition to the CT scans, we follow patients with radiographs 3 months after surgery, radiographs and tomograms 6 months after surgery, and radiographs 12 months after surgery. All patients were provided informed consent and use of the CT images was conducted with the approval of our institutional ethical committee.

We distinguished occult fractures from periprosthetic fractures found during surgery, defining occult fractures as those that could be confirmed only on the postoperative CT images, but were not seen intraoperatively or on postoperative radiographs. We reconstituted pre- and postoperative CT images performed 1 week after surgery using a helical CT scanner (LightSpeed VCT; GE Medical Systems, Milwaukee, WI, USA) and the AquariusNET ${ }^{\mathrm{TM}}$ program (TeraRecon Inc, San Mateo, CA, USA), with 1 mm slice thickness and $2.5 \mathrm{~mm}$ pitch for axial, sagittal, and coronal images. A fracture was diagnosed when a fracture line was confirmed in two or more planes on the reconstructed images (Fig. 2). The demographic data of patients were blinded when we diagnosed the fractures; although we could not discern the cup design used on the CT images, it could be surmised from the stem design noted on the CT scan. One of the authors $(\mathrm{KH})$ screened CT images of 486 hips, and other authors (TK, YK, DI) reviewed CT images of the detected occult fractures.

Patients with periacetabular fractures recognized during surgery were allowed nonweightbearing ambulation on the affected side for 3 weeks, and patients with periprosthetic occult acetabular fractures observed on the CT scans 1 

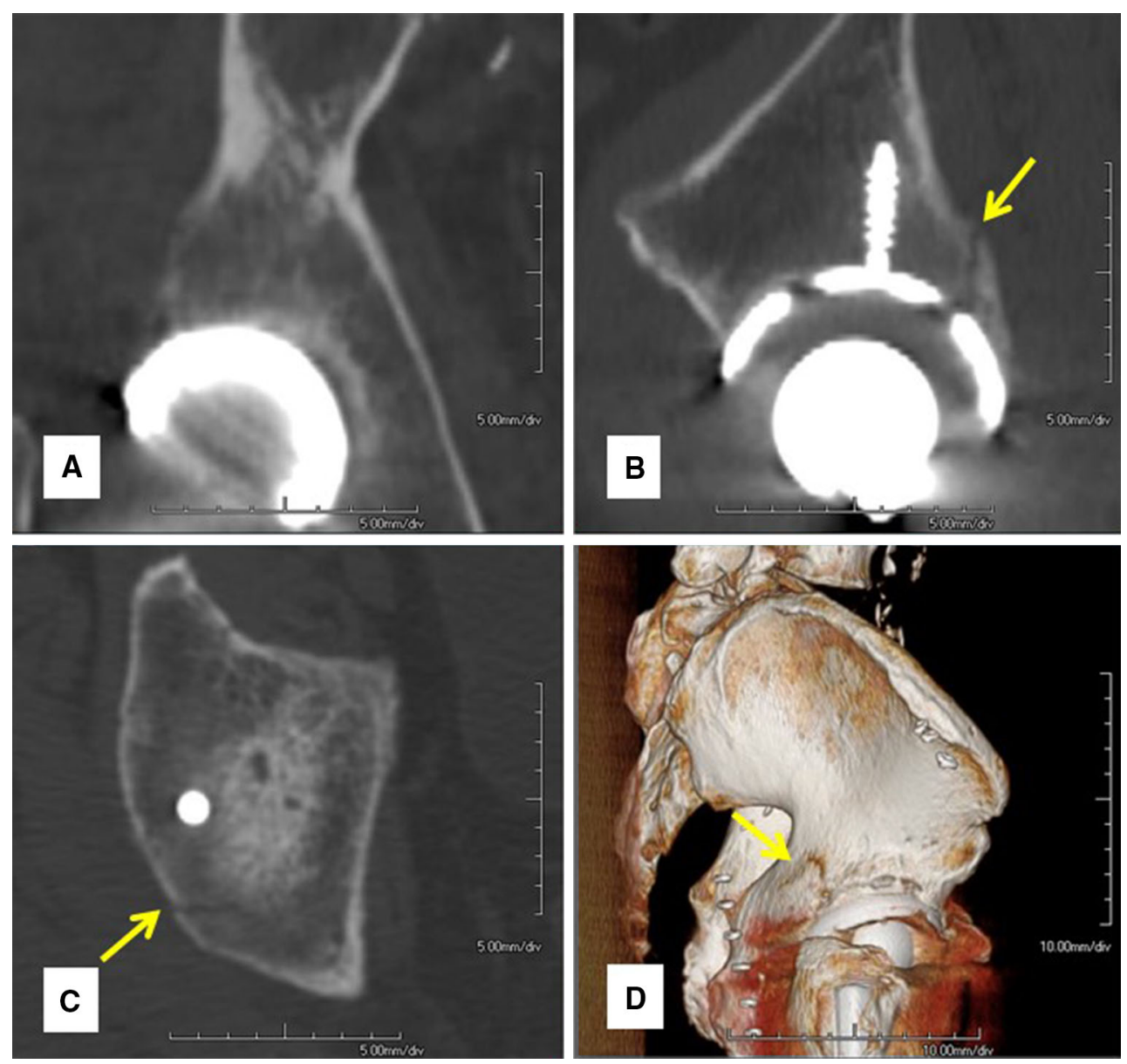

Fig. 2A-D We defined occult fractures as those that could be confirmed on the postoperative CT images but were not seen intraoperatively or found on postoperative radiographs. We reconstituted pre- and postoperative CT images (approximately 1 week after surgery) to (A) coronal, (B) sagittal, and (C) axial images and

diagnosed an occult fracture when we were able to confirm a fracture (arrow) line in two or more planes on the images. (D) We were able to confirm a fracture (arrow) line in the three-dimensional image.

week postoperatively began full weightbearing ambulation on the day after surgery with no alteration of their routine.

The statistical analyses defined a probability of 0.05 or less as significant on the univariate analysis using a chisquare test and Welch's t-test. The multivariate analysis used logistic regression analysis (SPSS Version 19.0; IBM Corp, Armonk, NY, USA) for analysis and likewise defined statistical significance as a probability of 0.05 or less.

\section{Results}

Occult fractures of the acetabulum were found in 41 of 486 hips $(8.4 \%)$. In two of 486 hips $(0.4 \%)$, periprosthetic fractures of the acetabulum were found during surgery.

The superolateral wall was the most frequent location for occult fractures of the acetabulum (Fig. 3). The superolateral wall was the site of fracture in 15 hips $(15 / 41$ hips; 37\%), the medial wall in eight (eight of 41;20\%), anterior wall in seven (seven of $41 ; 17 \%$ ), posterior wall in three (three of $41 ; 7 \%$ ), and other locations in eight hips (eight of $41 ; 20 \%$ ).

After controlling for the potentially relevant confounding variables of sex, age, BMI, additional dome screw fixation, cup composition (titanium alloy compared with $\mathrm{CoCr}$ ) and size of each cup, use of peripheral self-locking cups, and presence of rapidly destructive coxarthropathy (factors identified as potentially predictive in univariate analysis), only the use of peripheral self-locking cups was associated with increased risk of occult periprosthetic acetabular fracture (odds ratio [OR], 2.3 compared with hemispheric cups; 95\% CI, 1.2-5.6; $\mathrm{p}<0.05$ ) (Table 1). Of 486 acetabular cups, 377 were fixed using a press-fit technique with additional screw fixation, and of these, 32 (9\%) showed an occult fracture. The remaining 109 cups were fixed using press-fit only, and occult fractures were 
Fig. 3A-T (A) The diagram shows an occult fracture in the medial wall. CT reconstruction shows the occult fracture (arrow) line on the (B) axial, (C) sagittal, and (D) coronal images. (E) This diagram shows an occult fracture on the posterior wall. CT reconstruction shows the occult fracture (arrow) line on (F) axial, (G) sagittal, and (H) coronal images. (I) An occult fracture on the superolateral wall is shown. CT reconstruction shows the occult fracture (arrow) line on (J) axial, (K) sagittal, and (L) coronal images. (M) An occult fracture on the anterior wall is shown. CT reconstruction shows the occult fracture (arrow) line on the $(\mathbf{N})$ axial, $(\mathbf{O})$ sagittal, and (P) coronal images. (Q) An occult fracture at the area classified as "other location" is shown. The CT reconstruction shows the occult fracture (arrow) line on (R) axial, (S) sagittal, and (T) coronal image.
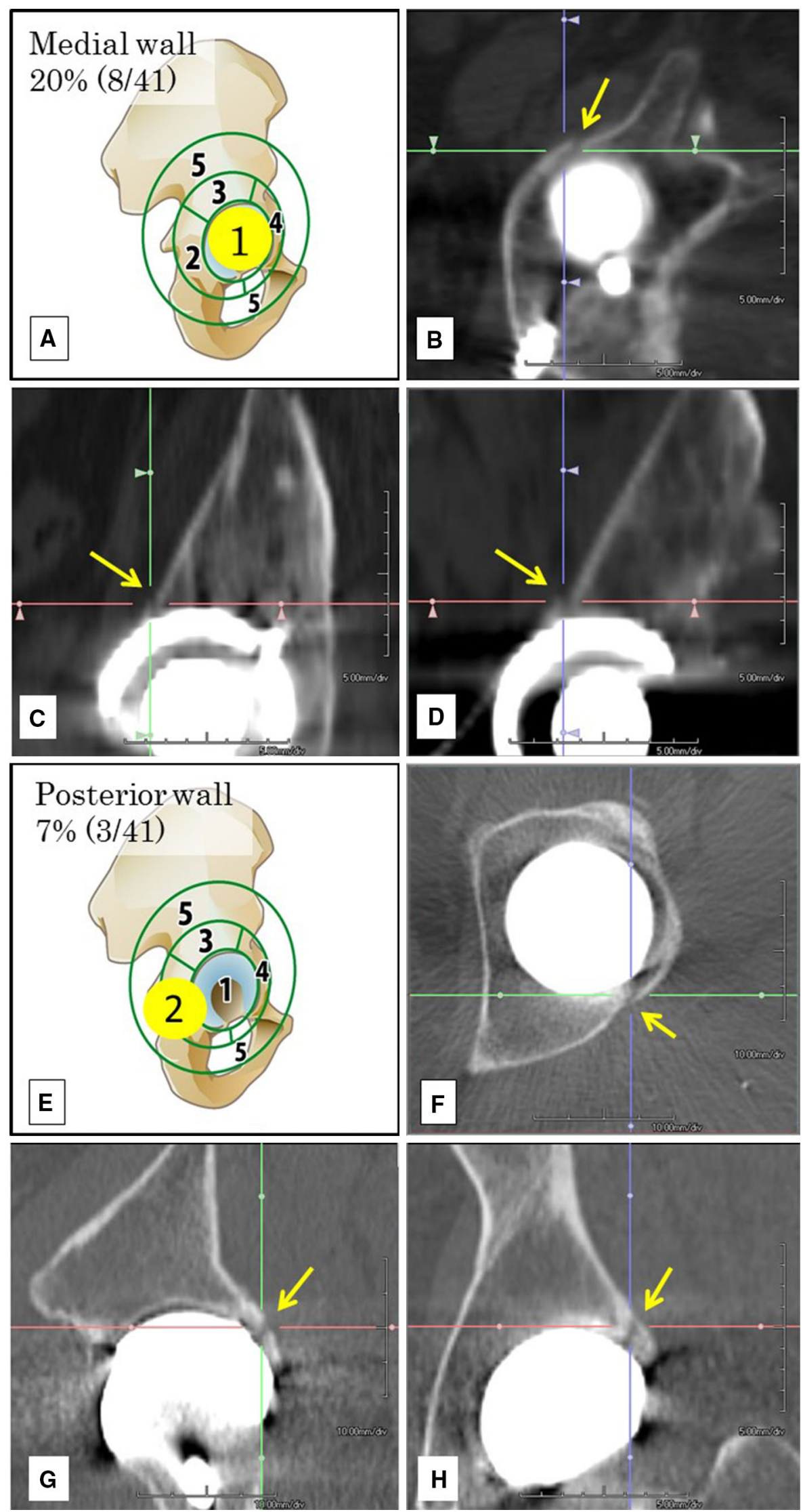
Fig. 3A-T continued
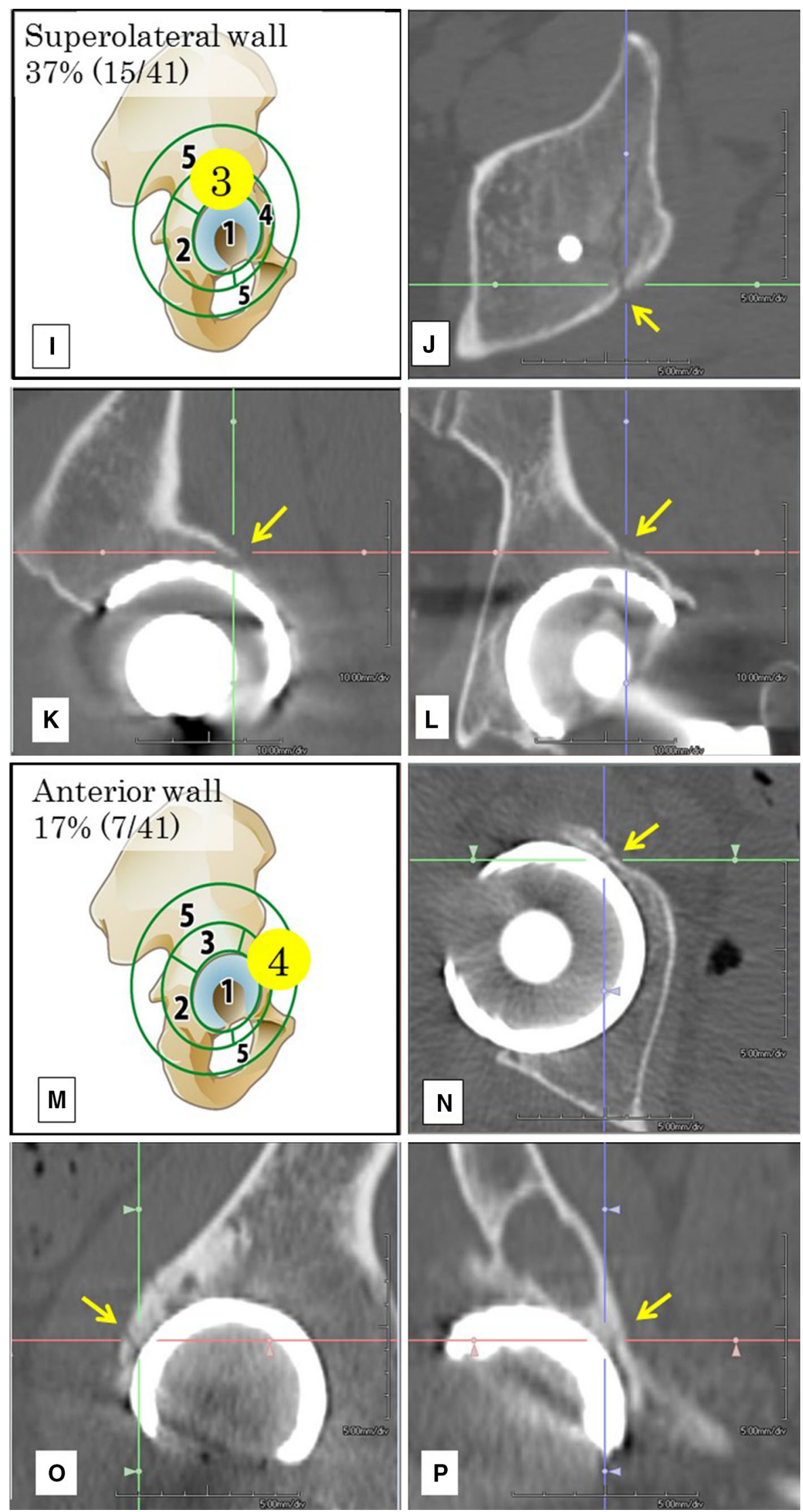
Fig. 3A-T continued
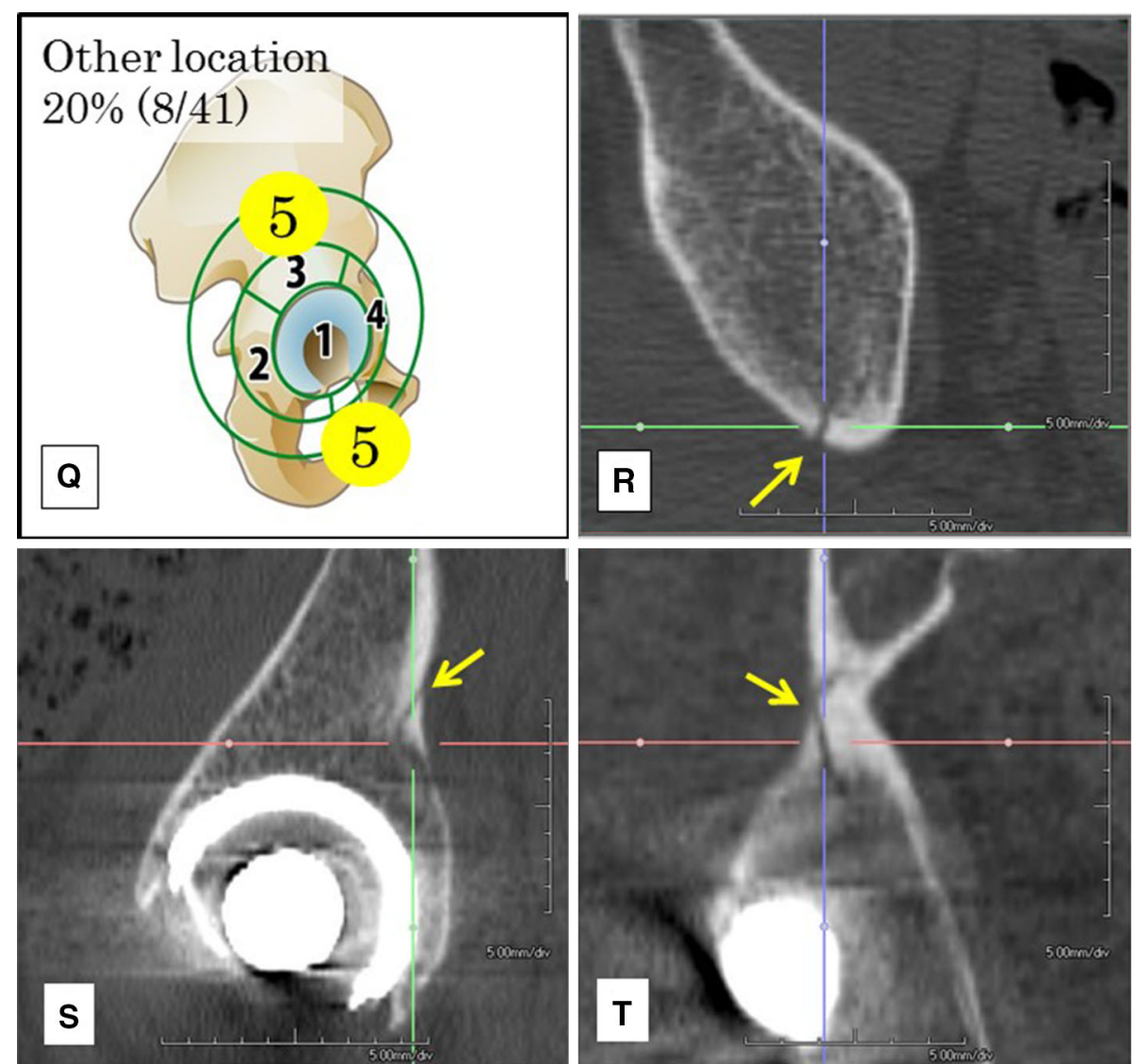

Table 1. Multivariate analysis of risk factors for periprosthetic fracture of the acetabulum during THA, including occult fractures

\begin{tabular}{|c|c|c|c|c|c|c|}
\hline \multirow[t]{2}{*}{ Variable } & \multicolumn{6}{|l|}{ Regression } \\
\hline & Coefficient (B) & SE & Wald statistic & Odds ratio & $\mathrm{p}$ Value & $95 \% \mathrm{CI}$ \\
\hline Sex & 0.02 & 0.02 & 0.91 & 1.02 & 0.34 & $0.30-1.24$ \\
\hline Age & -0.05 & 0.02 & 0.01 & 0.96 & 0.92 & $0.98-1.04$ \\
\hline BMI & 0.01 & 0.04 & 0.07 & 1.01 & 0.79 & $0.88-1.01$ \\
\hline PSL cup & 0.96 & 0.4 & 6.04 & 2.62 & $0.01 *$ & $1.07-4.74$ \\
\hline RDC & 0.46 & 0.8 & 0.30 & 1.58 & 0.59 & $0.33-8.54$ \\
\hline Cup size & 0.05 & 0.6 & 0.84 & 1.05 & 0.36 & $0.94-1.17$ \\
\hline Cup composition & 0.10 & 0.5 & 0.44 & 1.10 & 0.83 & $0.44-2.77$ \\
\hline Screw fixation & -0.44 & 0.4 & 1.04 & 0.64 & 0.30 & $0.27-1.50$ \\
\hline
\end{tabular}

SE = standard error; PSL = peripheral self-locking cup; RDC = rapidly destructive coxarthropathy; Cup composition = titanium alloy; screw fixation $=$ additional dome screw fixation; Cox-Snell R Square, 0.23; Nagelkerke R Square, 0.52; *p $<0.05$.

found in 11 of 109 (10\%) of these. A hemispheric cup (Trilogy ${ }^{\circledR}$ ) and a peripheral self-locking cup (Trident ${ }^{\circledR}$ PSL ${ }^{\circledR}$ HA) were used in the two patients for whom periprosthetic fractures of the acetabulum were found during surgery. In the patients with occult fractures, we used peripheral self-locking cups in 30 hips and hemispheric cups in 11 hips.

\section{Discussion}

Periprosthetic fracture of the acetabulum occurring during primary THA is a rare complication. Periprosthetic occult fractures of the acetabulum, to our knowledge, have not been evaluated previously using preoperative and postoperative CT. We were interested to determine how often 
such occult fractures occurred because these fractures might be a source of unexplained groin pain after THA and are not typically recognized by intraoperative findings or postoperative radiographs. We found occult fractures of the acetabulum in 41 of 486 primary hips (8\%), and most frequently on the superolateral wall. The use of peripheral self-locking cups was associated with an increased risk of occult periprosthetic acetabular fracture.

This study has several limitations. First is the issue of selection bias. In our institution, although the peripheral self-locking cup was the preferred choice when a Stryker stem was used, the cup was switched to a hemispheric type when it was judged to be too rigid in an attempt at press fit. This procedure introduces a selection bias for cups. Therefore, the rate of fractures with the peripheral selflocking cup may be underestimated. The mean age of the patients in our study was approximately 59 years and thus the patients were relatively young. However, most candidates for THA are between 60 and 69 years old in the registry in Japan [1], and the median age of THA candidates in the multicenter study by Fujita et al. [9] also skewed younger (62 years; range, 23-89 years). This may have affected the incidence of fracture. Second, we did not have bone density data for each patient, and with a preponderance of female patients (male, 124 joints; female, 362 joints), osteoporosis might have resulted in a higherthan-average risk of fracture in this population. Third, because only one surgeon performed the operations in our study it may limit generalizability, but it eliminates issues associated with variation in surgical techniques for cup implantation. Fourth, measurement of the hip center of rotation is lacking. The effects of inclination of the pelvis were too large to assess based on radiographs and we did not measure these in the current study. We targeted primary THA in our study, and cups were placed at the position of the original acetabulum by using a navigation system at our institution. Fifth, there were fewer patients with rapidly destructive coxarthropathy or rheumatoid arthritis compared with those with OA, and because of these small numbers the statistical power may be considered insufficient to evaluate these diagnoses as risk factors for occult fracture.

In this study, the rate of occult fractures of the acetabulum seen on postoperative CT images was $8 \%$ (41 of 486 hips). Conversely, the rate of periprosthetic acetabular fractures found during surgery was $0.4 \%$ (two of $486 \mathrm{hips}$ ), similar to those reported previously for fractures identified intraoperatively [12]. The occult fracture prevalence was unexpectedly high, and we were able to confirm that these minor periprosthetic fractures occurred during press-fit fixation. Because the periacetabular area has a complicated 3-D shape, the bone is cancellous, and the region is covered by dense soft tissue, it can be difficult to detect a fracture by perioperative macroscopic evaluation and on postoperative plain radiographs. However, special software (AquariusNET $^{\mathrm{TM}}$ ) with CT-DICOM images can easily produce excellent quality multiplanar reformations and reconstructed axial images around the acetabulum at any chosen section thickness, which enabled us to identify small cortical or cancellous fractures that could not be seen on postoperative radiographs or by direct evaluation during surgery. Sharkey et al. [25] reported that intraoperative fractures of the acetabulum occurred owing to insertion of an oversized acetabular component in an underreamed socket. They suggested that bone quality may be a significant factor and that sclerotic unyielding bone may be predisposed to fracture with underreaming; however, osteoporosis is likely a predisposing factor as well. In our study, sex, age, and presence of rheumatoid arthritis were not risk factors for periprosthetic occult fractures, so we cannot comment on their suggestions.

We found occult fractures in the medial wall and other locations such as the ilium and pubis, therefore to cover all fractures, we modified earlier classification schemes to five areas: (1) medial wall, (2) posterior wall, (3) superolateral wall, (4) anterior wall, and (5) other locations (Fig. 4). With this modified classification, the superolateral wall was the most frequent location (Fig. 3). The classification described by Callaghan et al. $[4,5]$ included four areas: (1) anterior wall, (2) transverse, (3) inferior lip, and (4) posterior wall, but did not allow for some of the locations that we detected. We found that fractures occurring mainly in the superolateral area are characteristic of occult fractures.

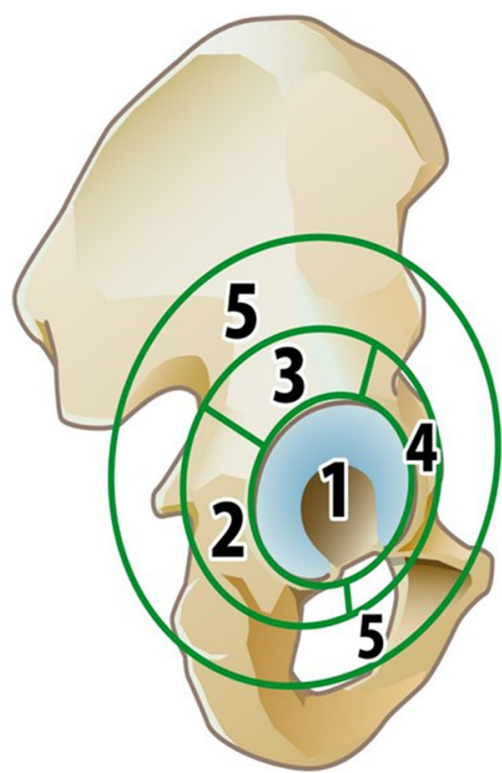

Fig. 4 The locations of occult fractures are classified in five areasthe (1) medial wall, (2) posterior wall, (3) superolateral wall, (4) anterior wall, and (5) other locations. 
Fig. 5A-H CT reconstruction shows the scans for a 64-yearold man with osteonecrosis of the femoral head. A peripheral self-locking cup with $1.8-\mathrm{mm}$ underreaming was used for the rim diameter. A periprosthetic occult fracture (arrow) of the acetabulum was seen on postoperative CT images. CT reconstruction shows the occult fracture (arrow) line on (A) coronal, (B) sagittal, (C) axial and (D) three-dimensional images. The occult fractures that had achieved bone union (arrow) 3 months later is seen on these (E) coronal, (F) sagittal, (G) axial, and (H) threedimensional $\mathrm{CT}$ reconstruction images. The implant had bone ingrowth with no malalignment. The patient had no additional treatment.
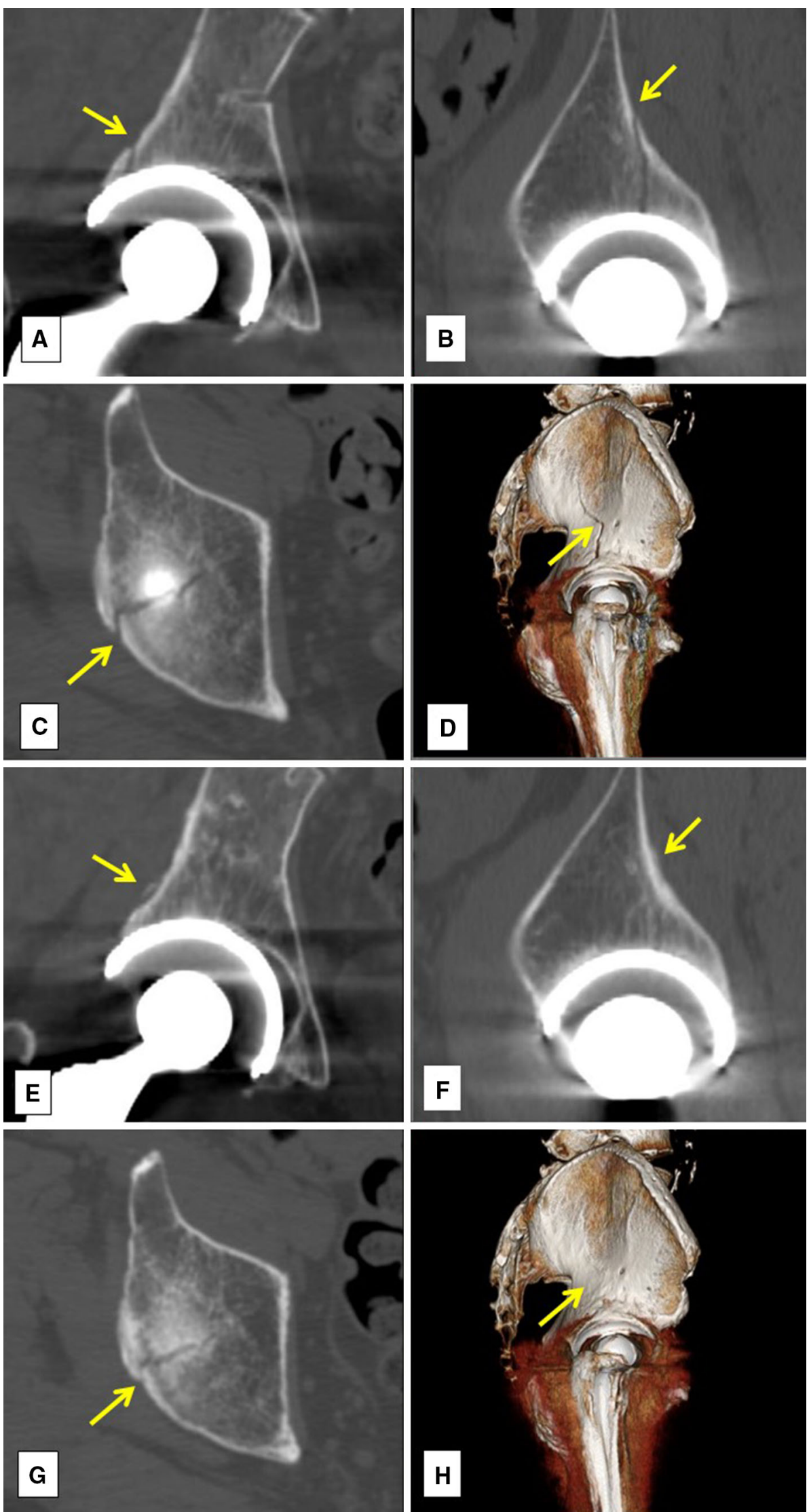
However, the fracture site did not affect treatment or prognosis. The bone quality of the superolateral wall is usually dense or sclerotic, and mechanical stress during press-fit fixation may concentrate in this location. We believe that occult fractures may occur even in the case of acetabular bone stock that is not particularly weak. Antoniades et al. [2] reported that the higher seating force associated with the peripheral self-locking design may result in an increased risk of intraoperative acetabular fractures in high-density bone stock.

After controlling for relevant confounding variables such as sex, age, BMI, additional dome screw fixation, the composition (titanium alloy compared with $\mathrm{CoCr}$ ), size of the cup, use of peripheral self-locking cups, and presence of rapidly destructive coxarthropathy, the use of peripheral self-locking cups was the only risk factor for periprosthetic fractures of the acetabulum, including occult fractures. In hips with peripheral self-locking cups, the acetabular bed was reamed to $1.8 \mathrm{~mm}$ smaller than the true largest external diameter of the component to be inserted. The bone quality around the acetabular rim is usually dense and sometimes very sclerotic, so this area may experience stress concentration during cup impaction. Haidukewych et al. [12] reported a high rate of intraoperatively detected periprosthetic fractures in association with the use of monoblock elliptical components. Previous reports have noted that inappropriate reaming size is a risk factor for periprosthetic fractures of the acetabulum [7, 19, 20, 25]. We had fewer occult fractures with hemispheric cups than with peripheral self-locking cups; all hemispheric cups were press-fit after 1 to $2 \mathrm{~mm}$ underreaming according to the patient's bone quality. Changing the reaming diameter according to the bone quality might result in a lower incidence of occult fractures with hemispheric cups. The peripheral self-locking cup was the first choice when we used the Trident ${ }^{\mathbb{R}}$ cup with Stryker femoral stems. The cup was switched to a hemispheric type when it was judged to be too rigid in an attempted impaction trial. As a result, the incidence of fractures with the peripheral self-locking cup may be underestimated. However, this switch occurred only in a small percentage of cases in which the peripheral selflocking cup was used. The composition and rigidity of cups also are considered to affect occult fractures in press-fit cases [12], but it was not a significant factor in our analysis.

The patients with occult fractures in our study did not receive additional treatment. As a treatment for intraoperative acetabular fractures, Helfet and Ali [13] recommended screw or plate fixation depending on the stability of the acetabular prosthesis. Postoperative fractures with clinically and radiographically stable implants were recommended to be treated with 6 to 8 weeks of toe-touch weightbearing exercise $[8,13]$. The two periprosthetic fractures we found during surgery were minor rim fractures, therefore the patients with these fractures were treated with additional dome screw fixation and were nonweightbearing for 3 weeks. At our institution, we intraoperatively confirm sufficient fixation of a cup by confirming the rigidity of press-fit and using more screws when the fixation is uncertain owing to the bone quality; therefore, we obtain sufficient fixation of a cup in cases of occult fracture as well. We believe union was achieved in all of the periprosthetic fractures found during surgery and for the occult fractures. Radiographic examinations at 12 months after surgery did not show any migration or alignment change in a cup, nor a circumferential radiolucent line in any periprosthetic fracture or occult fracture that occurred intraoperatively. Based on the radiographic signs for detecting acetabular osseointegration described by Moore et al. [22], all cases had either a superolateral buttress or inferomedial buttress, or both; therefore, we determined there was bony ingrowth fixation in all cases. A total of 38 of $43(88 \%)$ patients were followed for more than 3 years and 24 of $43(56 \%)$ patients were followed for more 5 years. Radiographic examinations at the last followup showed bony ingrowth fixation in all 43 cases of periprosthetic fractures of acetabulum. We also performed later followup CTs for some patients which showed fracture healing (Fig. 5). Although the occult fractures did not cause clinical issues in our patients, it is possible that such fractures may be a source of unexplained pain during the early postoperative period after THA.

We used CT to diagnose periprosthetic occult fractures of the acetabulum occurring during primary THA. Although the prevalence of intraoperative occult fractures of the acetabulum was $8.4 \%$, patients with these fractures did not require additional treatment. Peripheral self-locking cups may increase the risk of such periprosthetic occult fractures. We believe that fracture risk can be reduced by choosing the correct cup design, based on intraoperative findings. It is important to recognize that periprosthetic occult fractures may occur during press-fit impaction and to routinely confirm intraoperatively that the cup fixation is rigid.

\section{References}

1. Akiyama H, Hoshino A, Iida H, Shindo H, Takakura Y, Miura H, Yamamoto K, Yoshiya S, Hasegawa Y, Shimamura T, Kurosaka M, Otsuka H, Kawanabe K, Kawate K, Harada Y, Nakamura T; Implant Committee, Japanese Orthopaedic Association. A pilot project for the Japan arthroplasty register. J Orthop Sci. 2012;17:358-369.

2. Antoniades G, Smith EJ, Deakin AH, Wearing SC, Sarungi M. Primary stability of two uncemented acetabular components of different geometry: hemispherical or peripherally enhanced? Bone Joint Res. 2013;2:264-269. 
3. Berry DJ. Epidemiology: hip and knee. Orthop Clin North Am. 1999;30:183-190.

4. Callaghan JJ. Instructional Course Lectures, The American Academy of Orthopaedic surgeons: Periprosthetic fractures of the acetabulum during and following total hip arthroplasty. $J$ Bone Joint Surg Am. 1997;79:1416-1421.

5. Callaghan JJ, Kim YS, Pedersen DR, Brown TD. Periprosthetic fractures of the acetabulum. Orthop Clin North Am. 1999;30:221-234.

6. Chitre A, Wynn Jones H, Shah N, Clayson A. Complications of total hip arthroplasty: periprosthetic fractures of the acetabulum. Curr Rev Musculoskelet Med. 2013;6:357-363.

7. Curtis MJ, Jinnah RH, Wilson VD, Hungerford DS. The initial stability of uncemented acetabular components. J Bone Joint Surg Br. 1992;74:372-376.

8. Della Valle CJ, Momberger NG, Paprosky WG. Periprosthetic fractures of the acetabulum associated with a total hip arthroplasty. Instr Course Lect. 2003;52:281-290.

9. Fujita H, Katayama N, Iwase T, Otsuka H. Multi-center study of use of the Exeter stem in Japan: evaluation of 1000 primary THA. J Orthop Sci. 2012;17:370-376.

10. Fujita K, Kabata T, Maeda T, Kajino Y, Iwai S, Kuroda K, Hasegawa K, Tsuchiya $\mathrm{H}$. The use of the transverse acetabular ligament in total hip replacement: an analysis of the orientation of the trial acetabular component using a navigation system. Bone Joint J. 2014;96:306-311.

11. García-Rey E, García-Cimbrelo E, Cruz-Pardos A. Cup press fit in uncemented THA depends on sex, acetabular shape, and surgical technique. Clin Orthop Relat Res. 2012;470:3014-3023.

12. Haidukewych GJ, Jacofsky DJ, Hanssen AD, Lewallen DG. Intraoperative fractures of the acetabulum during primary total hip arthroplasty. J Bone Joint Surg Am. 2006;88:1952-1956.

13. Helfet DL, Ali A. Periprosthetic fractures of the acetabulum. Instr Course Lect. 2004;53:93-98.

14. Inoue D, Kabata T, Maeda T, Kajino Y, Fujita K, Hasegawa K, Yamamoto T, Takagi T, Ohmori T, Tsuchiya H. Usefullness of three-dimensional templating software to quantify the contact state between implant and femur in total hip arthroplasty. Eur $J$ Orthop Surg Traumatol. 2015;25:1293-1300.

15. Inoue D, Kabata T, Maeda T, Kajino Y, Fujita K, Hasegawa K, Yamamoto T, Tsuchiya H. Value of computed tomography-based three-dimensional surgical preoperative planning software in total hip arthroplasty with developmental dysplasia of the hip. J Orthop Sci. 2015;20:340-346.

16. Inoue D, Kabata T, Maeda T, Kajino Y, Yamamoto T, Takagi T, Ohmori $\mathrm{T}$, Tsuchiya $\mathrm{H}$. The correlation between clinical radiological outcome and contact state of implant and femur using three-dimensional templating software in cementless total hip arthroplasty. Eur J Orthop Surg Traumatol. 2016;26:591-598.
17. Jensen JS, Retpen JB. Failures with the Judet noncemented total hip. Acta Orthop Scand. 1987;58:23-26.

18. Kajino Y, Kabata T, Maeda T, Iwai S, Kuroda K, Tsuchiya H. Does degree of the pelvic deformity affect the accuracy of computed tomography-based hip navigation? J Arthroplasty. 2012;27:1651-1657.

19. Kim YS, Callaghan JJ, Ahn PB, Brown TD. Fracture of the acetabulum during insertion of an oversized hemispherical component. J Bone Joint Surg Am. 1995;77:111-117.

20. MacKenzie JR, Callaghan JJ, Pedersen DR, Brown TD. Areas of contact and extent of gaps with implantation of oversized acetabular components in total hip arthroplasty. Clin Orthop Relat Res. 1994;298:127-136.

21. Mayle RE, Della Valle CJ. Intra-operative fractures during THA: see it before it sees us. J Bone Joint Surg Br. 2012;94(11 suppl A):26-31.

22. Moore MS, McAuley JP, Young AM, Engh CA Sr. Radiographic signs of osseointegration in porous-coated acetabular components. Clin Orthop Relat Res. 2006;444:176-183.

23. Peterson CA, Lewallen DG. Periprosthetic fracture of the acetabulum after total hip arthroplasty. J Bone Joint Surg Am. 1996;78:1206-1213.

24. Schwartz JT Jr, Mayer JG, Engh CA. Femoral fracture during non-cemented total hip arthroplasty. J Bone Joint Surg Am. 1989;71:1135-1142.

25. Sharkey PF, Hozack WJ, Callaghan JJ, Kim YS, Hanssen AD, Lewallen DG. Acetabular fracture associated with cementless acetabular component insertion: a report of 13 cases. J Arthroplasty. 1999;14:426-431.

26. Sugano N, Nishii T, Nakata K, Masuhara K, Takaoka K. Polyethylene sockets and alumina ceramic heads in cemented total hip arthroplasty: a ten-year study. $J$ Bone Joint Surg Br. 1995;77:548-556.

27. Taylor MM, Meyers MH, Harvey JP Jr. Intraoperative femur fractures during total hip replacement. Clin Orthop Relat Res. 1978;137:96-103.

28. Toossi N, Adeli B, Timperley AJ, Haddad FS, Maltenfort M, Parvizi J. Acetabular components in total hip arthroplasty: is there evidence that cementless fixation is better? J Bone Joint Surg Am. 2013;95:168-174.

29. Troelsen A, Malchau E, Sillesen N, Malchau H. A review of current fixation use and registry outcomes in total hip arthroplasty: the uncemented paradox. Clin Orthop Relat Res. 2013;471:2052-2059.

30. Zwartelé RE, Witjes S, Doets HC, Stijnen T, Pöll RG. Cementless total hip arthroplasty in rheumatoid arthritis: a systematic review of the literature. Arch Orthop Trauma Surg. 2012;132:535-546. 